Pacific Journal of Mathematics

ON A CONJECTURE OF L. B. PAGE 


\section{ON A CONJECTURE OF L. B. PAGE}

\section{F. SCHUBERT}

If $\mathscr{K}$ is an Hilbert space and $\mathscr{K}$ a subspace which is invariant under a unilateral shift $S$ on $\mathscr{C}$ one can ask when a bounded operator $T$ on $\mathscr{M}$ which commutes with $S$ can be extended to a bounded operator on all of $\mathscr{K}$ which also commutes with $S$. Here this problem is considered in the special case that $\mathscr{K}$ is a Hardy space $H^{2}$ of functions analytic in the unit-disk with values in a finite dimensional Hilbert space. For this situation an easily derived necessary condition is shown to be sufficient. Further those $\mathscr{M}$ for which the extension to $\mathscr{K}$ is unique are characterized.

The problem posed above has recently been considered by Lavon B. Page [4], who conjectured that the condition (2) below was sufficient as well as necessary and who proved the validity of this conjecture in several cases.

Throughout this note we shall use facts, mainly elementary or standard, about unilateral shifts with few direct references given. These facts can all be found in [1.2.3]. A subspace will alwas be a closed linear manifold and all operators will be linear and continuous.

Let $\mathscr{K}_{n}$ denote the Hardy space of $H^{2}$ functions in the unit disk $\Delta=\{z|| z \mid<1\}$ with values in the complex Hilbert space $C_{n},(n<+$ $\infty)$. When convenient $u \in \mathscr{K}_{n}$ will be considered as an $n \times 1$ column vector of elements in $\mathscr{K}_{1}=H^{2}$, and we shall freely identify $\mathscr{K}_{p} \oplus$ $\mathscr{K}_{q}$ with $\mathscr{K}_{p+q}$. Let $S$ denote the unilateral shift on $\mathscr{K}_{n}$ generated by multiplication by $z$, i.e., $(S u)(z)=z u(z)$ for all $z \in \Delta$ and $u \in \mathscr{K}_{n}$. Then $S^{*} S=I$ but $S S^{*} \neq I$. For each positive integer $m$ put $P_{m}=$ $I-S^{m} S^{* m}$. Then $P_{m}$ is an orthogonal projection and for any operator $T$ commuting with $S, P_{m} T=P_{m} T P_{m}$. Thus for all $u \in \mathscr{K}_{n}$ we have,

$$
\left\|P_{m} T u\right\|=\left\|P_{m} T P_{m} u\right\| \leqq\|T\|\left\|P_{m} u\right\| .
$$

If $\mathscr{C}$ is a subspace of $\mathscr{K}_{n}$ which is invariant under $S$ and $T$ is an operator on $\mathscr{M}$ which commutes with $S$ and has a continuous extension to all of $\mathscr{K}_{n}$ which also commutes with $S$, then clearly this extension must satisfy (1) on $\mathscr{K}_{n}$. Hence $T$ itself must satisfy (1) on $\mathscr{A}$. This lead Page to the following conjecture [4]:

If $\mathscr{M}$ is a subspace of $\mathscr{K}_{n}$, invariant under $S$, and $T$ an operator on $\mathscr{M}$ which commutes with $S$ and satisfies (2) $\left\|P_{m} T u\right\| \leqq \alpha\left\|P_{m} u\right\|$ for all $u \in \mathscr{L}$ and $m$, then $T$ has an extension to all of $\mathscr{K}_{n}$ which commutes with $S$ and has norm less than or equal to $\alpha$.

We have taken the liberty here of reducing Page's conjecture 
for general $\mathscr{K}$ and $S$ to our situation. The purpose of this note is to prove the validity of the above conjecture. As our methods are essentially finite dimensional matrix arguments we are unable to prove Page's original conjecture. However some slight extensions are possible (see Remark 2.1). We can also decide whether or not an extension is unique.

1. Existence. If $\mathscr{C}$ is a subspace of $\mathscr{K}_{n}$, invariant under $S$, then $\mathscr{C}$ may be represented as $A \mathscr{K}_{p}$, for some $p \leqq n$, where $A$ is an isometry and $A S=S A$. Further $A$ can be represented as an $n \times$ $p$ matrix $A(z)$ of elements in $H^{\infty}$ with the further property that $A\left(e^{i \theta}\right)$ is an isometry in $C_{p}$, i.e., $\bar{A}^{\prime}\left(e^{i \theta}\right) A\left(e^{i \theta}\right)=I_{p}$, a.e. $0 \leqq \theta<2 \pi$. Here $\bar{A}^{\prime}\left(e^{i \theta}\right)$ is the complex conjugate transpose of the matrix $A\left(e^{i \theta}\right)$. In this representation $A$ acts on $u \in \mathscr{K}_{p}$ by matrix multiplication, $(A u)(z)=A(z) u(z)$ for all $z \in \Delta . A(z)$ is uniquely determined by $\mathscr{C}$ to within multiplication on the right by a constant unitary matrix and rank $A(z)=p$ except at a discrete set of points in $\Delta$.

If $T$ is an operator on $\mathscr{C}$ which commutes with $S$, then there exists an operator $G$ on $\mathscr{K}_{p}$, with $\|G\|=\|T\|$, defined uniquely by the equation

$$
T A u=A G u \text { for all } u \in \mathscr{K}_{p} \text {. }
$$

Moreover $G$ commutes with $S$ and so is representable as a $p \times p$ matrix of elements of $H^{\infty}$. We base our extension procedure on $G$.

In order to reduce the inequality (2) to a less intrinsic but more computible form, let $\mathscr{L}_{n}$ denote the space of $L^{2}$ functions on the unit circle with values in $C_{n}$. $\mathscr{K}_{n}$ is a closed subspace of $\mathscr{L}_{n}$. If $P_{0}$ denotes orthogonal projection of $\mathscr{L}_{n}$ onto the orthogonal complement of $\mathscr{K}_{n}$, then $\left(P_{0} u\right)\left(e^{i \theta}\right)$ is that part of the Fourier series for $u \in \mathscr{L}_{n}$ which contains only the negative powers of $e^{i \theta}$. We use $U$ to denote the minimal unitary extension of $S$ to $\mathscr{L}_{n}$, i.e. $U$ is the bilateral shift $(U u)\left(e^{i \theta}\right)=e^{i \theta} u\left(e^{i \theta}\right)$. Put $\hat{\mathscr{L}}=\left\{u \in \mathscr{L}_{n} \mid u\left(e^{i \theta}\right)=A\left(e^{i \theta}\right) v\left(e^{i \theta}\right), v \in \mathscr{L}_{p}\right\}$. Then $\hat{\mathscr{C}}$ is a bilaterally invariant subspace which contains $\mathscr{M}$. It is easily seen to be the closure in $\mathscr{L}_{n}$ of $\left\{u \in \mathscr{L}_{n} \mid U^{m} u \in \mathscr{C}\right.$ for some integer $m \geqq 0\}$. $T$ can also be extended to $\hat{T}$ on all of $\hat{\mathscr{C}}$ so that $\hat{T} U=U \hat{T}$ as $(\hat{T} u)\left(e^{i \theta}\right)=A\left(e^{i \theta}\right) G\left(e^{i \theta}\right) v\left(e^{i \theta}\right)$ for all $u=A v \in \hat{\mathscr{R}}$. The above extensions can of course be carried out more abstractly but there seems little point in doing so here.

LEMma 2.1. If $T$ is defined on $\mathscr{M}$, commutes with $S$ and satisfies (2), then

$$
\left\|P_{0} \hat{T} u\right\| \leqq \alpha\left\|P_{0} u\right\| \text { for all } u \in \mathcal{L}_{\mathcal{C}}
$$


Proof. Suppose $u \in \hat{\mathscr{C}}$ is such that $U^{m} u \in \hat{\mathscr{C}}$ for some integer $m$. Then, since $T$ satisfies (2),

$$
\begin{aligned}
\left\|P_{0} \hat{T} u\right\| & =\left\|P_{m} U^{m} \hat{T} u\right\|=\left\|P_{m} T U^{m} u\right\| \\
& \leqq \alpha\left\|P_{m} U^{m} u\right\|=\alpha\left\|P_{0} u\right\| .
\end{aligned}
$$

Since such $u \in \hat{\mathscr{C}}$ are dense, (3) holds by continuity for all $u \in \hat{\mathscr{L}}$. In particular if $z \in \Delta$ and $X \in C_{p}$ are arbitrary, then $v=X\left[e^{i \theta}-z\right]^{-1} \epsilon$ $\mathscr{L}_{p}$ and $A v \in \hat{\mathscr{L}}$. A short computation gives $\left(P_{0} A v\right)\left(e^{i \theta}\right)=A(z) v\left(e^{i \theta}\right)$ and $\left(P_{0} T A v\right)\left(e^{i \theta}\right)=(T A)(z) v\left(e^{i \theta}\right)=A(z) G(z) v\left(e^{i \theta}\right)$. Substitution of these identities into (3) yields (4) immediately.

THEOREM 2.1. Let $\mathscr{H}$ be a subspace of $\mathscr{K}_{n}$ which is invariant under $S$. Let $T$ be an operator on $\mathscr{M}$ which commutes with $S$ and satisfies (2). Then $T$ has an extension $T_{1}$ to all of $\mathscr{K}_{n}$ which commutes with $S$ and satisfies $\left\|T_{1}\right\| \leqq \alpha$.

Proof. Following Lemma 2.1, $\|A(z) G(z) X\| \leqq \alpha\|A(z) X\|$ for all $z \in \Delta$ and $X \in C_{p}$. Since $A(z)$ has rank $p$ except on a discrete subset of $\Delta$, there exists a $p \times p$ submatrix of $A(z)$ whose determinant vanishes on a discrete subset $D$ of $\Delta$. Without loss of generality this matrix may be taken as the first $p$ rows of $A(z)$. Extend $A(z), G(z)$, to $n \times n$ matrices $A_{1}(z), G_{1}(z)$ as below,

$$
A_{1}(z)=\left[\begin{array}{l:l} 
& 0 \\
A(z) & \\
& I_{n-p}
\end{array}\right], G_{1}(z)=\left[\begin{array}{l:r}
G(z) & 0 \\
\hdashline 0 & 0
\end{array}\right] \text {. }
$$

With $T_{1}(z)=A_{1}(z) G_{1}(z) A_{1}^{-1}(z)$ for $z \in \Delta-D$, we have $\left\|T_{1}(z) X\right\| \leqq \alpha\|X\|$ for all $X$ in the range of $A_{1}(z)=C_{n}$ and all $z \in \Delta-D$. For $Y \in C_{n}$, $F(z)=\bar{Y}^{\prime} T_{1}(z) X$ is analytic and satisfies $|F(z)| \leqq \alpha\|X\|\|Y\|$ for $z \epsilon$ $\Delta-D$. But $D$ is discrete and so $F(z)$ may be extended, by Riemann's Theorem on removable singularities, to all of $\Delta$ as an analytic funtion. Since $X, Y$ are arbitrary we have finally that $T_{1}(z)$ can be extended analytically to all of $\Delta$ and and satisfies there $\left\|T_{1}(z) X\right\| \leqq \alpha\|X\|$ for all $X \in C_{n}$. Now $T_{1}(z) A_{1}(z) X=A_{1}(z) G_{1}(z) X=(T A)(z) X$ for all $X \epsilon$ $C_{p}$ and $z \in \Delta$. Thus $T_{1}$ on $\mathscr{K}_{n}$ defined by $\left(T_{1} u\right)(z)=T_{1}(z) u(z)$ is an extension of $T$ which clearly commutes with $S$ and $\left\|T_{1}\right\| \leqq \alpha$.

REMARK 2.1. The above theorem covers the general case of unilateral shifts of finite multiplicity. By minor modifications the proof will also be valid for unilateral shifts $S$ on $\mathscr{K}$ of infinite multiplicity 
provided $(S \mathscr{C})^{\perp}$ contains a finite dimensional subspace $M$ such that $u(z) \in M$ for all $z \in \Delta$ and $u \in \mathscr{M}$.

3. Uniqueness. It follows immediately from (1) and (2) that the extension $T_{1}$ of Theorem 2.1 is an extension of minimal norm. However it need not be the only extension. Clearly an extension will be unique if and only if $\mathscr{C l}$ is not contained in the kernel of any non-trivial operator on $\mathscr{K}_{n}$ which commutes with $S$. The following lemma provides the necessary characterization in terms of the subspace $\mathscr{C}_{z}=\{u(z) \mid u \in \mathscr{M}\}$ of $C_{n}$, for each $z \in \Delta$.

Lemma 2.1. If $\mathscr{l} \subset \mathscr{K}_{n}$ is invariant under $S$, then $\mathscr{L}$ is contained in the kernel of a non-trivial operator $B$ on $\mathscr{K}_{n}$ which commutes with $S$ if and only if $\operatorname{dim} \mathscr{C l}_{z}<n$ for all $z \in \Delta$.

Proof. Suppose there exists an operator $B \neq 0$ on $\mathscr{L}_{n}$ such that $B S=S B$ and $B \mathscr{C}=0$. The corresponding matrix $B(z)$ is not identically zero and so vanishes at most on a discrete subset of $\Delta$. Since $B(z) \mathscr{C}_{z}=0$ we must have $\operatorname{dim} \mathscr{H}_{z}<n$ except possibly on a discrete subset of $\Delta$. However $\mathscr{C}=A \mathscr{K}_{p}$ and so $\operatorname{dim} \mathscr{C}_{z} \leqq p$ with strict inequality only on a discrete subset of $\Delta$. Thus $p<n$ and $\operatorname{dim} \mathscr{C}_{z}<n$ for all $\boldsymbol{z}$.

Conversely, if $\operatorname{dim} \mathscr{C}_{z}<n$ for all $z \in \Delta$ then $p<n$ in the representation of $\mathscr{l}$. With $A_{1}(z)$ the extension of $A(z)$ used in Theorem 2.1 , put $B_{1}(z)=\operatorname{det} A_{1}(z) A_{1}^{-1}(z)$, i.e., the matrix of co-factors of $A_{1}(z)$ in transposed position. Then the elements of $B_{1}(z)$ are in $H^{\infty}$ and if $B(z)$ denotes the $n \times n$ matrix whose first $p$ rows are zero and whose last $n-p$ rows are the last rows of $A_{1}(z)$, we have $B(z) A(z) \equiv 0$. The continuous operator $B$ on $\mathscr{K}_{n}$ defined by $B(z)$ then satisfies $B \mathscr{C l}=0$.

Corollary 3.1. If $T$ on $\mathscr{I l}$ has an extension to all of $\mathscr{K}_{n}$ which commutes with $S$ then the extension is unique if and only if dim $\mathscr{H}_{z}=n$ for some $z \in \Delta$.

Even if $\operatorname{dim} \mathscr{C}_{z}=n$ for all $z \in \Delta, T$ need not have any extension to all of $\mathscr{K}_{n}$. Modifying Example 2.2 of [4] take $n=p=2, A:(u$, $v) \rightarrow(u, \varphi v)$ where $\varphi$ is a singular inner function and $G:(u, v) \rightarrow(v, 0)$. Then $T$ defined on $\mathscr{M}=A \mathscr{K}_{2}$ by $T A=A G$ is a continuous map of $\mathscr{C l}$ into itself which commutes with $S$. If $T_{1}$ is any continuous extension of $T$ to $\mathscr{K}_{2}$ which commutes with $S$ then also $\varphi T_{1}=T \varphi$. But if $w \neq 0$ is in $H^{2}$ and is orthogonal to $\varphi H^{2}$ then $T_{1} \varphi(0, w)=(w$, $0)$ which is orthogonal to $\varphi T_{1}(0, w)$. Thus no continuous extension exists even though $\operatorname{dim} \mathscr{C}_{z}=2$ for all $z \in \Delta$. 


\section{REFERENCES}

1. P. R. Halmos, A Hilbert space problem book, Van Nostrand, Princeton, N. J., 1967.

2. H. Helson, Lectures on invariant subspaces, Academic Press, New York, 1964.

3. P. D. Lax, Translation invariant spaces, Acta. Math., 101 (1959), 163-178.

4. Lavon B. Page, Operators that commute with a unilateral shift on an invariant subspace, Pacific J. Math., 36 (1971), 787-794.

Received August 9, 1971.

QueEn's University, Kingston, Ontario 



\section{PACIFIC JOURNAL OF MATHEMATICS}

\section{EDITORS}

\section{H. SAMELSON}

Stanford University

Stanford, California 94305

\section{R. HOBBY}

University of Washington

Seattle, Washington 98105

\section{J. DugundjI}

Department of Mathematics University of Southern California Los Angeles, California 90007

RICHARD ARENS

University of California

Los Angeles, California 90024

\section{ASSOCIATE EDITORS}
E. F. BECKENBACH
B. H. NeumanN
F. WOLF
K. YOSHIDA

\section{SUPPORTING INSTITUTIONS}

\author{
UNIVERSITY OF BRITISH COLUMBIA \\ CALIFORNIA INSTITUTE OF TECHNOLOGY \\ UNIVERSITY OF CALIFORNIA \\ MONTANA STATE UNIVERSITY \\ UNIVERSITY OF NEVADA \\ NEW MEXICO STATE UNIVERSITY \\ OREGON STATE UNIVERSITY \\ UNIVERSITY OF OREGON \\ OSAKA UNIVERSITY
}

\author{
UNIVERSITY OF SOUTHERN CALIFORNIA \\ STANFORD UNIVERSITY \\ UNIVERSITY OF TOKYO \\ UNIVERSITY OF UTAH \\ WASHINGTON STATE UNIVERSITY \\ UNIVERSITY OF WASHINGTON \\ AMERICAN MATHEMATICAL SOCIETY \\ NAVAL WEAPONS CENTER
}

The Supporting Institutions listed above contribute to the cost of publication of this Journal, but they are not owners or publishers and have no responsibility for its content or policies.

Mathematical papers intended for publication in the Pacific Journal of Mathematics should be in typed form or offset-reproduced, (not dittoed), double spaced with large margins. Underline Greek letters in red, German in green, and script in blue. The first paragraph or two must be capable of being used separately as a synopsis of the entire paper. The editorial "we" must not be used in the synopsis, and items of the bibliography should not be cited there unless absolutely necessary, in which case they must be identified by author and Journal, rather than by item number. Manuscripts, in duplicate if possible, may be sent to any one of the four editors. Please classify according to the scheme of Math. Rev. Index to Vol. 39. All other communications to the editors should be addressed to the managing editor, Richard Arens, University of California, Los Angeles, California, 90024.

50 reprints are provided free for each article; additional copies may be obtained at cost in multiples of 50 .

The Pacific Journal of Mathematics is published monthly. Effective with Volume 16 the price per volume (3 numbers) is $\$ 8.00$; single issues, $\$ 3.00$. Special price for current issues to individual faculty members of supporting institutions and to individual members of the American Mathematical Society: $\$ 4.00$ per volume; single issues $\$ 1.50$. Back numbers are available.

Subscriptions, orders for back numbers, and changes of address should be sent to Pacific Journal of Mathematics, 103 Highland Boulevard, Berkeley, California, 94708.

PUBLISHED BY PACIFIC JOURNAL OF MATHEMATICS, A NON-PROFIT CORPORATION

Printed at Kokusai Bunken Insatsusha (International Academic Printing Co., Ltd.), 270, 3-chome Totsuka-cho, Shinjuku-ku, Tokyo 160, Japan. 


\section{Pacific Journal of Mathematics}

\section{Vol. 42, No. $3 \quad$ March, 1972}

Catherine Bandle, Extensions of an inequality by Pólya and Schiffer for vibrating membranes ................................ 543

S. J. Bernau, Topologies on structure spaces of lattice groups.......... 557

Woodrow Wilson Bledsoe and Charles Edward Wilks, On Borel product measures .......................................

Eggert Briem and Murali Rao, Normpreserving extensions in subspaces of

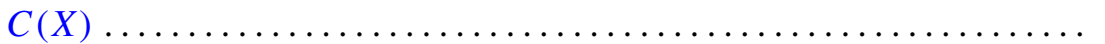

Alan Seymour Cover, Generalized continuation.................. 589

Larry Jean Cummings, Transformations of symmetric tensors .......... 603

Peter Michael Curran, Cohomology of finitely presented groups .......... 615

James B. Derr and N. P. Mukherjee, Generalized quasicenter and

hyperquasicenter of a finite group ...................... 621

Erik Maurice Ellentuck, Universal cosimple isols .................. 629

Benny Dan Evans, Boundary respecting maps of 3-mainfolds .......... 639

David F. Fraser, A probabilistic method for the rate of convergence to the

Dirichlet problem .................................. 657

Raymond Taylor Hoobler, Cohomology in the finite topology and Brauer

groups ..................................... 667

Louis Roberts Hunt, Locally holomorphic sets and the Levi form ........ 681

B. T. Y. Kwee, On absolute de la Vallée Poussin summability............ 689

Gérard Lallement, On nilpotency and residual finiteness in semigroups .... 693

George Edward Lang, Evaluation subgroups of factor spaces........... 701

Andy R. Magid, A separably closed ring with nonzero torsion pic ....... 711

Billy E. Rhoades, Commutants of some Hausdorff matrices ............. 715

Maxwell Alexander Rosenlicht, Canonical forms for local derivations . . . . 721

Cedric Felix Schubert, On a conjecture of L. B. Page ................ 733

Reinhard Schultz, Composition constructions on diffeomorphisms of $S^{p} \times S^{q}$

J. P. Singhal and H. M. (Hari Mohan) Srivastava, A class of bilateral generating functions for certain classical polynomials ....

Richard Alan Slocum, Using brick partitionings to establish conditions which insure that a Peano continuum is a 2-cell, a 2-sphere or an annulus...

James F. Smith, The p-classes of an $H^{*}$-algebra ...

Jack Williamson, Meromorphic functions with negative zeros and positive

poles and a theorem of Teichmuller ................. 